\title{
The Moon Illusion Explained by Wundt Illusion
}

\author{
Ahmad Yousef $^{1}$ \\ ${ }^{1}$ School of Computational Science and Engineering, McMaster University, Hamilton, Ontario, Canada \\ *Correspondence: mohamas2@mcmaster.ca
}

The Moon illusion is an optical illusion that causes the moon to appear much bigger near the horizon. Noticeably, the moon near the horizon is further than the moon higher up in the sky from the frames of references (humans) though.

Many scientists think that Ebbinghaus illusion may explain the moon illusion, and this interpretation might resolve some scientific debates. I, however, think that there are other physical and psychophysical theories that may also explain the moon illusion. One of these theories is, Wundt 'verticalhorizontal' illusion, as shown below. The theory states that a vertical line, of two perpendicular equal lines, appears to be much longer than the horizontal one, more specifically $30 \%$ longer.
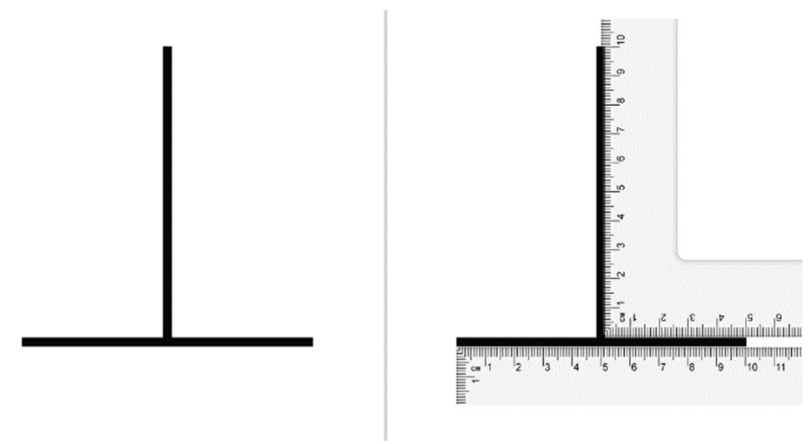

Now, imagine that you can draw two virtual lines from your position, as frame of reference, to the moon, for two different conditions: when the moon higher up in the sky; and when it's near the horizon. According to Wundt illusion, the horizontal line will appear shorter than the vertical one, and thus your brain will automatically understand that the 'near horizon' moon is much closer. Your brain will therefore perceive the moon near the horizon, to be much bigger, to fully comply to Wundt effect. I personally think that Wundt effect might not only be a perceptual illusion created by the physiological processes of human visual system, but also, it might explain a part of undetectable/undefined physics. These undetectable physics along with the detectable ones, I think, are interweaved together in a greater fabric to eventually create human visual awareness as a part of human global consciousness.

\section{Transactional References}

[1] Restle, Frank (1970). "Moon Illusion Explained on the Basis of Relative Size". Science.

[2] Robinson, J.O. (1998). The psychology of visual illusion. Courier Dover Publications.

[3] Wundt W. (1858). "Vertical-horizontal illusion", Published online Wikipedia, 2015.

[4] Yousef, Ahmad. 2019. "Consciousness Might Be Localized in Extra Physical Dimensions." PsyArXiv. June 11. doi:10.31234/osf.io/angc8. 